\title{
PAPER
}

\section{Acetylcholinesterase inhibition: a novel approach in the treatment of neurogenic orthostatic hypotension}

\author{
W Singer, T L Opfer-Gehrking, B R McPhee, M J Hilz, A E Bharucha, P A Low
}

See Editorial Commentary, p 1187

J Neurol Neurosurg Psychiatry 2003;74:1294-1298

See end of article for authors' affiliations

......................

Correspondence to: Dr Phillip A Low,

Department of Neurology, Mayo Clinic, 811

Guggenheim, 200 First

Street SW, Rochester, MN

55905, USA;

low@mayo.edu

Received 5 August 2002

In revised form

29 January 2003

Accepted 9 March 2003
Background: Pharmacological treatment of orthostatic hypotension is often limited because of troublesome supine hypertension.

Objective: To investigate a novel approach to treatment using acetylcholinesterase inhibition, based on the theory that enhanced sympathetic ganglion transmission increases systemic resistance in proportion to orthostatic needs.

Design: Prospective open label single dose trial.

Material: 15 patients with neurogenic orthostatic hypotension caused by: multiple system atrophy $(n=7)$, Parkinson's disease $(n=3)$, diabetic neuropathy $(n=1)$, amyloid neuropathy $(n=1)$, and idiopathic autonomic neuropathy $(\mathrm{n}=3)$.

Methods: Heart rate, blood pressure, peripheral resistance index (PRI), cardiac index, stroke index, and end diastolic index were monitored continuously during supine rest and head up tilt before and one hour after an oral dose of $60 \mathrm{mg}$ pyridostigmine.

Results: There was only a modest non-significant increase in supine blood pressure and PRI. In contrast, acetylcholinesterase inhibition significantly increased orthostatic blood pressure and PRI and reduced the fall in blood pressure during head up tilt. Orthostatic heart rate was reduced after the treatment. The improvement in orthostatic blood pressure was associated with a significant improvement in orthostatic symptoms.

Conclusions: Acetylcholinesterase inhibition appears effective in the treatment of neurogenic orthostatic hypotension. Orthostatic symptoms and orthostatic blood pressure are improved, with only modest effects in the supine position. This novel approach may form an alternative or supplemental tool in the treatment of orthostatic hypotension, specially for patients with a high supine blood pressure.
M aintenance of normotension in the standing position poses a substantial challenge for the cardiovascular system. Under normal circumstances, a significant fall in blood pressure can be prevented by a complex regulatory system comprising a series of neurohumoral mechanisms and cardiovascular reflexes, regulating vascular resistance, capacitance, stroke volume, and heart rate, with blood pressure as the controlled variable. ${ }^{1-3}$

Orthostatic hypotension is defined as a fall in blood pressure of at least $20 \mathrm{~mm} \mathrm{Hg}$ systolic or $10 \mathrm{~mm} \mathrm{Hg}$ diastolic within three minutes in the upright position. ${ }^{4}$ Patients with symptomatic orthostatic hypotension complain of lightheadedness, weakness, faintness, visual blurring, and impaired cognition in the upright position, resolving when sitting or lying down. ${ }^{5}$

In most patients, orthostatic hypotension is of neurogenic origin, resulting from impaired cardiovascular adrenergic function. The lesion can be postganglionic, as in pure autonomic failure and the autonomic neuropathies, or preganglionic, as in multiple system atrophy.

A major problem of current pharmacotherapeutic options is the non-selective elevation of blood pressure regardless of the body position, with supine hypertension as a common, troublesome, and typically dose limiting side effect. ${ }^{6-10}$ We hypothesised that as ganglionic transmission is low in the supine position and is greatly increased during orthostasis, enhancing ganglionic transmission by inhibiting acetylcholinesterase might selectively reduce orthostatic hypotension without causing significant supine hypertension. ${ }^{11}{ }^{12}$ Our specific aim in this prospective study was therefore to evaluate the efficacy and characteristics of acetylcholinesterase inhibition in the treatment of neurogenic orthostatic hypotension.

\section{METHODS}

\section{Patient selection}

Patients with neurogenic orthostatic hypotension were selected on the basis of the following criteria.

\section{Inclusion criteria}

- Adult men or women aged over 18 years.

- Orthostatic blood pressure fall of at least $20 \mathrm{~mm} \mathrm{Hg}$ systolic or $10 \mathrm{~mm} \mathrm{Hg}$ diastolic within three minutes of a $70^{\circ}$ head up tilt.

- Presence of symptoms including lightheadedness, weakness, faintness, and blurred vision in the upright position, resolving when lying down.

- Neurogenic origin of orthostatic hypotension determined by medical history, general clinical examination, and clinical autonomic evaluation.

- A score of at least 3 on a 10 point composite autonomic severity scale (CASS), which is based on standardised autonomic reflex testing and is normalised for age and $\operatorname{sex}^{13-16}$ : four points are allotted for cardiovascular adrenergic failure and three points each for sudomotor and cardiovagal failure.

\section{Exclusion criteria}

- Pregnant or lactating women.

- The presence of another cause of orthostatic hypotension.

- Failure of other organ systems or systemic illness that can affect the study results, autonomic function, or the patient's ability to cooperate, including congestive heart failure, significant coronary artery disease, arrhythmia, renal disease, severe anaemia, hypothyroidism, and cerebrovascular accidents. 
Table 1 Patient characteristics

\begin{tabular}{|c|c|c|c|c|c|c|c|c|}
\hline Patient No & Diagnosis & $\begin{array}{l}\text { Age } \\
\text { (years) }\end{array}$ & Sex & CASS $_{\text {sudo }}$ & CASS $_{\text {vagal }}$ & CASS $_{\text {adren }}$ & $\mathrm{CASS}_{\text {total }}$ & $\begin{array}{l}\text { Duration of } \\
\mathrm{OH} \text { (months) }\end{array}$ \\
\hline 1 & Multiple system atrophy & 63 & $\mathrm{~F}$ & 0 & 1 & 4 & 5 & 35 \\
\hline 2 & Multiple system atrophy & 56 & $M$ & 1 & 1 & 2 & 4 & 16 \\
\hline 3 & Multiple system atrophy & 76 & $\mathrm{~F}$ & 3 & 3 & 4 & 10 & 10 \\
\hline 4 & Multiple system atrophy & 47 & $M$ & 0 & 1 & 3 & 4 & 18 \\
\hline 5 & Multiple system atrophy & 63 & $M$ & 0 & 3 & 2 & 5 & $>60$ \\
\hline 6 & Multiple system atrophy & 67 & $\mathrm{~F}$ & 1 & 0 & 2 & 3 & 36 \\
\hline 7 & Multiple system atrophy & 72 & $M$ & 2 & 2 & 3 & 7 & 30 \\
\hline 8 & Parkinson's disease with AF & 68 & $M$ & 0 & 3 & 2 & 5 & 15 \\
\hline 9 & Parkinson's disease with AF & 70 & $\mathrm{~F}$ & 2 & 1 & 3 & 6 & 50 \\
\hline 10 & Parkinson's disease with $\mathrm{AF}$ & 69 & $\mathrm{~F}$ & 2 & 0 & 2 & 4 & 50 \\
\hline 11 & Diabetic autonomic neuropathy & 56 & $\mathrm{~F}$ & 3 & 3 & 4 & 10 & 48 \\
\hline 12 & Amyloid neuropathy & 65 & $M$ & 2 & 2 & 4 & 8 & 3 \\
\hline 13 & Idiopathic autonomic neuropathy & 18 & M & 3 & 1 & 2 & 6 & 32 \\
\hline 14 & Idiopathic autonomic neuropathy & 31 & $M$ & 3 & 0 & 2 & 5 & 19 \\
\hline 15 & Idiopathic autonomic neuropathy & 21 & $\mathrm{~F}$ & 0 & 1 & 2 & 3 & $>60$ \\
\hline
\end{tabular}

$\mathrm{AF}$, autonomic failure; $\mathrm{CASS}_{\text {adren, }}$ composite autonomic severity scale, adrenergic function; $\mathrm{CASS}_{\text {sudo, }}$ composite autonomic severity scale, sudomotor

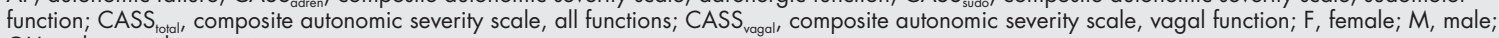
$\mathrm{OH}$, orthostatic hypotension.

- Concomitant treatment with anticholinergic agents, adrenergic antagonists, vasoactive agents, or other drugs that could interfere with testing of autonomic function; if such a drug had to be discontinued, patients were off treatment for at least five half lives.

\section{Study design and treatment}

The study was a prospective open label trial of the efficacy of a single oral dose of $60 \mathrm{mg}$ pyridostigmine (Mestinon ${ }^{\mathrm{TM}}$ ) in the treatment of neurogenic orthostatic hypotension. The study protocol was approved by the institutional review board and was carried out in accordance with the Declaration of Helsinki (1989) of the World Medical Association. All subjects gave their written informed consent. Fifteen patients recruited from the Mayo outpatient clinic participated in the study. Recordings were started in the morning between 09.00 and 11.00 hours, at least two hours after a light breakfast, at controlled ambient room temperature $\left(23^{\circ} \mathrm{C}\right)$. No caffeine or nicotine was allowed for one day before the study.

After 30 minutes of rest in the supine position, patients were tilted to a $70^{\circ}$ upright position for five minutes and then returned to the supine position for another five minutes. Head up tilt was interrupted only if presyncopal symptoms or a fall in systolic blood pressure below $60 \mathrm{~mm} \mathrm{Hg}$ occurred.

The protocol was repeated one hour after drug administration. Immediately after completion of the recordings, patients were asked to scale their orthostatic symptoms on a visual analogue scale from 0 to 10 for both tilts.

Primary end points were the influence of acetylcholinesterase inhibition on supine and orthostatic blood pressure and on orthostatic symptoms; secondary end points were its influence on other cardiovascular indices; predictors of a positive response to treatment; and side effects.

\section{Data acquisition}

Photoplethysmographic determination of blood pressure

Beat to beat blood pressure was recorded continuously using the photoplethysmographic volume clamp method (Finapres model 2300, Ohmeda, Englewood, Colorado, USA). The analogue blood pressure signal was sampled at $250 \mathrm{~Hz}$ and the maximum and minimum points occurring between the QRS pulse derived as systolic blood pressure (SBP) and diastolic blood pressure (DBP). Mean blood pressure (MBP) was calculated using the formula: $\mathrm{MBP}=\mathrm{DBP}+(\mathrm{SBP}-\mathrm{DBP}) / 3$.

Recording of heart rate and calculation of vagal baroreflex sensitivity

Instantaneous heart rate was calculated from the RR interval using continuous three lead ECG recordings. Vagal baroreflex gain was derived from RR intervals and systolic blood pressure values during five minutes of supine baseline recording using the sequence method. ${ }^{16}{ }^{17}$

\section{Impedance cardiography}

Changes in thoracic impedance during the cardiac cycle reflect changes in thoracic fluid volume and velocity, the main contributor being the thoracic aorta. ${ }^{18}$ Impedance cardiography (BoMed NCCOM3 R-7, BoMed Medical Manufacturing, Irvine, California, USA) uses these dynamic changes of bioimpedance (sampled at $500 \mathrm{~Hz}$ ) together with ECG and blood pressure recordings for a beat to beat calculation of cardiac output, stroke volume, end diastolic volume, and total peripheral resistance; these are normalised by body surface area to cardiac index, stroke index, end diastolic index, and peripheral resistance index.

\section{Respiration}

Respiratory excursion was measured using a nasal thermistor. The respiratory signal was sampled at $250 \mathrm{~Hz}$ and the data point occurring coincident with the QRS pulse written to file.

\section{Data analysis}

Cardiovascular variables at baseline and during tilt, as well as changes with tilt, were analysed off-line by a data analyst blinded to the treatment status. In order to minimise the influence of signal oscillations, 30 second averages of data were generated. The last 30 seconds before tilt were defined as baseline, and 30 seconds around minute 3 of tilt were defined as the tilt value. Paired two tailed $t$ test was used for statistical analysis of cardiovascular variables before and after treatment. The Wilcoxon rank-sum test was used to compare symptoms. For the evaluation of relations between variables, we used linear regression analysis and Spearman's rank correlation.

All data are expressed as mean (SEM). Significance was accepted at the $5 \%$ level. Correction factors for multiple testing were not applied as the statistical interest greatly differed among comparisons. It was preferable to undertake multiple tests without corrections and observe the pattern of results. ${ }^{19}$

\section{RESULTS}

\section{Patient characteristics}

Demographic and clinical data, as well as findings of autonomic reflex testing (CASS scores), are summarised in table 1. Eight men and seven women participated. Their mean (SEM) age was $56.2(4.8)$ years. 
Table 2 Cardiovascular variables in the supine position and during head up tilt before and after pyridostigmine

\begin{tabular}{|c|c|c|c|c|}
\hline \multirow[b]{2}{*}{ Variable } & \multicolumn{2}{|c|}{ Before pyridostigmine } & \multicolumn{2}{|c|}{ After pyridostigmine } \\
\hline & Supine & Upright & Supine & Upright \\
\hline $\mathrm{SBP}(\mathrm{mm} \mathrm{Hg})$ & 155.5 (7.9) & $110.9(7.5)$ & $158.0(7.2)$ & $124.3(8.3)^{*} \dagger$ \\
\hline $\mathrm{MBP}(\mathrm{mm} \mathrm{Hg})$ & $98.4(3.7)$ & $75.8(4.0)$ & $102.0(3.5)$ & $85.5(4.9)^{*} \dagger$ \\
\hline $\mathrm{DBP}(\mathrm{mm} \mathrm{Hg})$ & $70.4(1.9)$ & $58.7(2.8)$ & $74.5(2.4)$ & $66.5(3.8)^{*} \dagger$ \\
\hline PRI (dyn.s. $\left.\mathrm{cm}^{-5} \cdot \mathrm{m}^{-2}\right)$ & 2361 (207) & $1774(173)$ & $2534(258)$ & $2181(170)^{*}$ \\
\hline HR (beats/min) & $75.5(3.4)$ & $95.6(7.6)$ & $72.6(3.2)$ & $87.8(5.8)^{*}$ \\
\hline $\mathrm{Cl}\left(1 / \mathrm{min} / \mathrm{m}^{2}\right)$ & $3.56(0.28)$ & $3.74(0.39)$ & $3.54(0.29)$ & $3.44(0.29)$ \\
\hline $\mathrm{SI}\left(\mathrm{ml} / \mathrm{m}^{2}\right)$ & $46.4(2.6)$ & $39.1(3.6)$ & $48.7(3.9)$ & $40.0(3.9)$ \\
\hline $\mathrm{EDI}\left(\mathrm{ml} / \mathrm{m}^{2}\right)$ & 73.2 (3.9) & $71.6(6.0)$ & $78.8(4.7)$ & 74.1 (6.7) \\
\hline \multicolumn{5}{|c|}{$\begin{array}{l}\text { Values are mean (SEM). } \\
\text { *Significant difference comparing variables for the same body position before and } \\
\text { after pyridostigmine. } \\
\text { †Significant difference comparing position induced changes before and after pyridostigmine. } \\
\mathrm{Cl} \text {, index for cardiac output; } \mathrm{DBP} \text {, diastolic blood pressure; EDI, index for end diastolic volume; HR, heart } \\
\text { rate; MBP, mean blood pressure; PRI, peripheral resistance index; SBP, systolic blood pressure, SI, index for } \\
\text { stroke volume. }\end{array}$} \\
\hline
\end{tabular}

Ten patients were diagnosed as having orthostatic hypotension of primary central origin (multiple system atrophy $(\mathrm{n}=7)$ and Parkinson's disease with autonomic failure $(\mathrm{n}=3))$; five had a peripheral cause (diabetic polyneuropathy $(\mathrm{n}=1)$, amyloid neuropathy $(\mathrm{n}=1)$, and idiopathic autonomic neuropathy $(\mathrm{n}=3))$. The CASS score was $5.7(0.6)$, suggesting moderate to severe autonomic failure.

\section{Cardiovascular recordings}

\section{Supine rest}

Baseline variables were not significantly different before and after pyridostigmine (table 2), though a moderate but non-significant increase in supine blood pressure was observed after the drug in some patients.
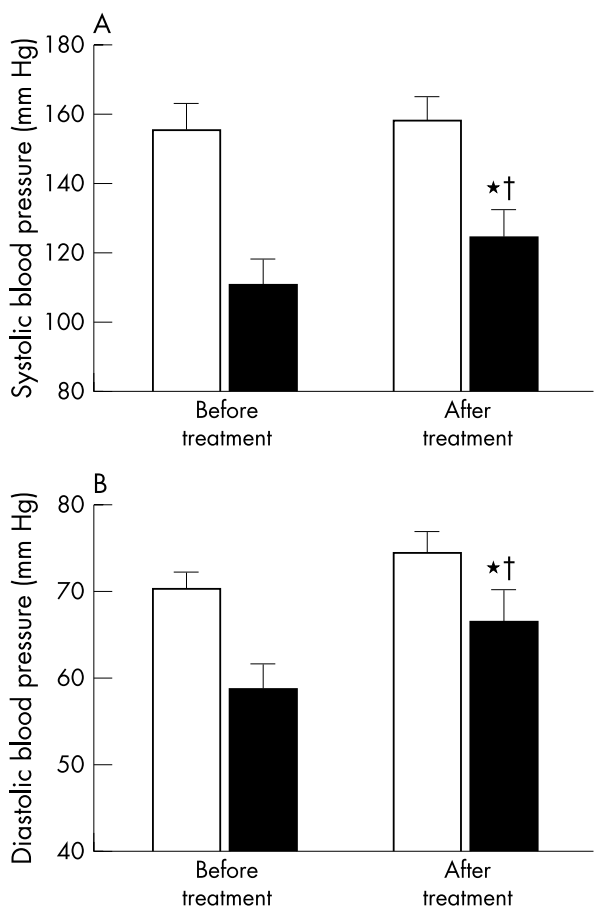

Figure 1 Systolic (A) and diastolic (B) blood pressure in the supine position (light bars) and during head up tilt (dark bars) before and after treatment with pyridostigmine. * Significant difference $(p<0.05)$ comparing variables for the same body position before and after pyridostigmine; $†$ significant difference $(p<0.05)$ comparing tilt induced changes before and after pyridostigmine.
Head up tilt

There was significant improvement in orthostatic blood pressure after pyridostigmine (table 2, fig 1). Peripheral resistance index in the upright position was also increased after pyridostigmine, while orthostatic heart rate was reduced. Cardiac index, stroke index, and end diastolic index did not change.

Changes with tilt

The blood pressure fall in the upright position was significantly attenuated after treatment with pyridostigmine (table 2, fig 1). There were no changes in heart rate, peripheral resistance index, cardiac index, stroke index, or end diastolic index after pyridostigmine.

\section{Orthostatic symptoms}

The improvement in orthostatic blood pressure was associated with amelioration of orthostatic symptoms (fig 2A). There was a significant correlation between symptomatic improvement and improvement in orthostatic blood pressure fall (fig 2B).

\section{Predictors of therapeutic response}

We sought to determine predictors of a good therapeutic response to acetylcholinesterase inhibition. No significant correlation was found between improvement in orthostatic blood pressure and the severity of cardiovascular adrenergic impairment $\left(\mathrm{CASS}_{\text {adren }}\right)$, overall autonomic failure $\left(\mathrm{CASS}_{\text {total }}\right)$, pretreatment blood pressure fall, or age. The response to the drug was also not significantly different in orthostatic hypotension of primary central origin compared with peripheral origin. However, there was a negative correlation between vagal baroreflex gain and reduction in the orthostatic blood pressure fall (fig 3).

\section{Side effects}

A 63 year old man with multiple system atrophy reported sweating and perspiration starting about 30 minutes after taking pyridostigmine, lasting for about one hour.

A 76 year old woman with multiple system atrophy reported modest urinary urgency 30 minutes after taking pyridostigmine. Whether or not the drug was the cause of this event remains uncertain. The patient had experienced similar symptoms before. Urodynamic studies had revealed neurogenic bladder dysfunction.

A 70 year old woman with Parkinson's disease and autonomic failure complained of modest abdominal cramping following a meal, five hours after taking pyridostigmine. Owing to the long latency between pyridostigmine intake and symptom occurrence, this problem could not be clearly attributed to the drug. 

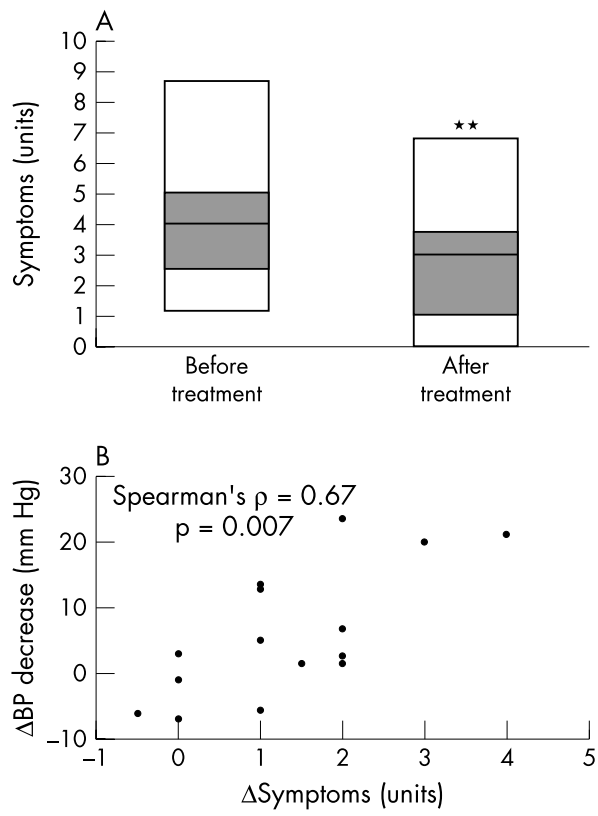

Figure 2 Orthostatic symptoms (10th, 25th, 50th, 75th, and 90th centile) before and after pyridostigmine treatment $(A)$, and the relation between improvement in orthostatic symptoms and improvement in orthostatic blood pressure decrease (B). $* *$ Significant difference $(p<0.01 v$ before treatment).

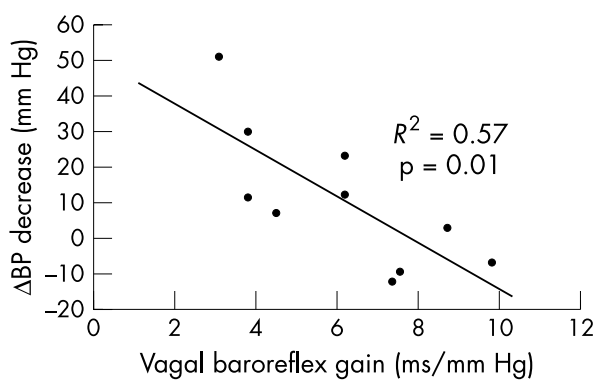

Figure 3 Relation between baroreflex sensitivity and improvement in orthostatic blood pressure fall.

No other side effects were reported.

\section{DISCUSSION}

Symptomatic treatment of orthostatic hypotension comprises non-pharmacological and pharmacological measures. Apart from sodium chloride in the form of salt tablets, pharmacological treatment is currently focused mainly on two drugs, fludrocortisone and midodrine, the efficacy of which has been demonstrated in several controlled clinical trials. ${ }^{6-9} 2021$

Supine hypertension is one of the most common side effects of these drugs, because the elevation of blood pressure is not selective for body position..$^{6-9}$ As supine hypertension is a common feature of orthostatic hypotension itself, it is also the most troublesome side effect, often limiting the value of these drugs. ${ }^{10}$

The baroreceptor reflex plays a key role in blood pressure homeostasis. A fall in blood pressure leads to unloading of baroreceptors located in the carotid sinus and aortic arch, resulting in reduced traffic in glossopharyngeal and vagal afferents, activation of sympathetic outflow, and inhibition of cardiovagal neurones. ${ }^{23-25}$

An ideal therapeutic concept of orthostatic hypotension would be to increase sympathetic outflow selectively in the standing position. A drug acting under baroreflex control would have minor effects in the supine position and would be

most effective when the patient is standing. We present the novel concept of enhancing sympathetic ganglionic transmission by inhibition of acetylcholinesterase. Inhibiting this enzyme facilitates the transmission of impulses from cholinergic neurones across the synaptic cleft. As the preganglionic sympathetic neurone is cholinergic and the traffic through sympathetic ganglia is modest in the supine position and greatly increases with standing, we sought to achieve adrenergic vasomotor effects proportional to orthostatic needs. ${ }^{11} 122627$

Our data suggest that oral administration of $60 \mathrm{mg}$ pyridostigmine is effective in the treatment of neurogenic orthostatic hypotension. Both standing blood pressure and peripheral resistance were significantly increased, while the orthostatic fall in blood pressure was attenuated. This improvement in blood pressure in the upright position was associated with significant amelioration of orthostatic symptoms. Furthermore, amelioration of orthostatic blood pressure and symptomatic improvement were significantly correlated.

A moderate increase in blood pressure in the supine position after pyridostigmine was observed in some patients, but overall, supine blood pressure was not significantly affected, supporting our theory of a baroreflex controlled drug action. Cook et al even described a lower resting diastolic blood pressure after pyridostigmine, ${ }^{28}$ while Gemmill et al reported largely unaltered supine blood pressures following edrophonium injection. ${ }^{29}$ This fairly selective effectiveness of pyridostigmine in the standing position may be its most beneficial characteristic, bearing in mind the problem of supine hypertension.

Cardiac output, stroke volume, and end diastolic volume were not significantly altered in the lying or upright position. Supine heart rate was only mildly lower after pyridostigmine, but orthostatic heart rate was significantly reduced. While it is well recognised that acetylcholinesterase inhibition can induce bradycardia, our findings suggest that this effect is heart rate dependent, supporting the findings of other investigators on temperature regulation and exercise after pyridostigmine administration, and on the influence of pyridostigmine on heart rate responses to mental stress. ${ }^{30-32}$ This phenomenon might be explained by the dominating direct effect of pyridostigmine on muscarinic synapses being more evident in the upright position when there is vagal withdrawal and sympathetic activation. ${ }^{30}$ This mechanism seems to exceed an indirect ganglionic enhancement of sympathetic outflow to the heart.

Our concept of selective enhancement of ganglionic neurotransmission involves afferent as well as efferent baroreceptor pathways. As there was no correlation between drug response and the severity of autonomic - and in particular adrenergic failure, we assume that the presence of only some preserved pathways is sufficient to improve vasomotor function significantly. Our findings of a negative correlation between baroreflex sensitivity and improvement in the fall in orthostatic blood pressure suggest that the response may even be better in patients with severely impaired baroreflex function.

Though published reports suggest a difference between central and peripheral autonomic failure in the supine noradrenaline response to acetylcholinesterase inhibition, supine blood pressure responses are not reported to differ significantly. ${ }^{29}$ While it is unclear whether such a difference in the noradrenaline response is also present in the upright position, we did not find a difference between primary central and peripheral autonomic failure in the orthostatic blood pressure response to pyridostigmine.

During this single dose trial, three patients (20\%) reported modest side effects following pyridostigmine intake, including perspiration, urinary urgency, and abdominal discomfort. However, in two instances the symptoms could not be clearly attributed to the drug, and it remains possible that they were purely coincidental. Side effects of pyridostigmine are most 
commonly related to overdosage and are mainly caused by activation of muscarinic receptors. Increased peristalsis resulting in abdominal cramps and diarrhoea is typically the most irritating side effect. ${ }^{33}{ }^{34}$ In most patients with neurogenic orthostatic hypotension, there is generalised autonomic failure, often including the gastrointestinal autonomic network. ${ }^{35-37}$ We would therefore expect activation of muscarinic receptors to be beneficial by improving the common symptom of constipation in this patient group rather than being a significant source of adverse side effects. ${ }^{38}$

\section{Conclusions}

Enhancement of ganglionic transmission by inhibition of acetylcholinesterase appears to be an effective and generally well tolerated method of treating neurogenic orthostatic hypotension. This novel approach could be of particular value for patients with supine hypertension. Further studies are needed to confirm the results of this open trial in a double blind protocol. It will be important to investigate potential side effects of long term treatment carefully in this patient group and to explore the optimal dose regimen.

Furthermore, it is unclear from the present data whether there might be a more pronounced effect on supine blood pressure under conditions of mental or physical stress. This should be addressed in future studies, as such an effect might limit the benefit of this new approach in clinical practice.

\section{Authors' affiliations}

W Singer, T L Opfer-Gehrking, B R McPhee, P A Low, Department of Neurology, Mayo Clinic, Rochester, Minnesota, USA

A E Bharucha, Department of Gastroenterology, Mayo Clinic M J Hilz, Department of Neurology, University of Erlangen-Nuremberg, Germany

\section{Competing interests: none declared}

\section{REFERENCES}

1 Joyner MJ, Shepherd JT. Autonomic regulation of circulation. In: Low PA, ed. Clinical autonomic disorders: evaluation and management, 2nd ed. Philadelphia: Lippincott-Raven, 1997:61-71.

2 Wieling W, van Lieshout JJ. Maintenance of postural normotension in humans. In: Low PA, ed. Clinical autonomic disorders: evaluation and management, 2nd ed. Philadelphia: Lippincott-Raven, 1997:73-82.

3 Smit AAJ, Halliwill JR, Low PA, et al. Pathophysiological basis of orthostatic hypotension in autonomic failure. J Physiol (Lond) 1999;519:1-10

4 Consensus statement. The definition of orthostatic hypotension, pure autonomic failure and multiple system atrophy. Clin Auton Res 1996:6:125-6.

5 Low PA, Opfer-Gehrking TL, McPhee BR, et al. Prospective evaluation of clinical characteristics of orthostatic hypotension. Mayo Clin Proc 1995;70:617-22.

6 Robertson D, Davis TL. Recent advances in the treatment of orthostatic hypotension. Neurology 1995:45(suppl 5):S26-32.

7 Chobanian AV, Volicer L, Tifft CP, et al. Mineralocorticoid-induced hypertension in patients with orthostatic hypotension. N Engl J Med 1979;301:68-73.

8 Low PA, Gilden JL, Freeman R, et al. Efficacy of midodrine vs placebo in neurogenic orthostatic hypotension. A randomized, double-blind multicenter study. Midodrine Study Group. JAMA 1997;277:1046-51.

9 Wright RA, Kaufmann HC, Perera R, et al. A double-blind dose-response study of midodrine in neurogenic orthostatic hypotension. Neurology 1998;51:120-4

10 Shannon J, Jordan J, Costa F, et al. The hypertension of autonomic failure and its treatment. Hypertension 1997;30:1062-7.
11 Wallin BG, Elam M. Microneurography and autonomic dysfunction. In: Low PA, ed. Clinical autonomic disorders: evaluation and management, 2nd ed. Philadelphia: Lippincott-Raven, 1997:233-43.

12 Thompson JM, Jennings GL, Chin JP, et al. Measurement of human sympathetic nervous responses to stressors by microneurography. J Auton Nerv Syst 1994:49:277-81.

13 Low PA. Autonomic nervous system function. J Clin Neurophysiol 1993; 10:14-27.

14 Low PA. Laboratory evaluation of autonomic function. In: Low PA, ed Clinical autonomic disorders: evaluation and management, 2 nd ed. Philadelphia: Lippincott-Raven, 1997:179-208.

15 Low PA. Composite autonomic scoring scale for laboratory quantification of generalized autonomic failure. Mayo Clin Proc 1993;68:748-52.

16 Fritsch JM, Eckberg DL, Graves LD, et al. Arterial pressure ramps provoke linear increases of heart period in humans. Am J Physiol 1986;251:R1086-90.

17 Rudas L, Crossman AA, Morillo CA, et al. Human sympathetic and vagal baroreflex responses to sequential nitroprusside and phenylephrine. Am J Physiol 1999;276:H1691-8.

18 Newman DG, Callister R. The non-invasive assessment of stroke volume and cardiac output by impedance cardiography: a review. Aviat Space Environ Med 1999:70:780-9.

19 O'Brien PC. The appropriateness of analysis of variance and multiple comparison procedures. Biometrics 1983;39:787-8

20 Fealey RD, Robertson D. Management of orthostatic hypotension. In: Low PA, ed. Clinical autonomic disorders: evaluation and management, 2nd ed. Philadelphia: Lippincott-Raven, 1997:763-75.

21 Polinsky RJ. Clinical autonomic neuropharmacology. Neurol Clin 1990:8:77-92.

22 Davies IB, Bannister RG, Sever PS, et al. Fludrocortisone in the treatmen of postural hypotension: altered sensitivity to pressor agents. $\mathrm{Br} J \mathrm{Clin}$ Pharmacol 1978;6:444-5P.

23 Benarroch EE. The central autonomic network. In: Low PA, ed. Clinical autonomic disorders: evaluation and management, 2 nd ed. Philadelphia: Lippincott-Raven, 1997: 17-23.

24 Loewy AD. Central autonomic pathways. In: Loewy AD, Spyer KM, eds. Central regulation of autonomic functions. New York: Oxford University Press, 1990:88-103.

25 Dampney RA. Functional organization of central pathways regulating the cardiovascular system [review]. Physiol Rev 1994;74:323-64.

26 McKinzie S, Tyce GM, Brimijoin S. Lowered norepinephrine turnover as a sign of impaired ganglionic transmission after preganglionic lesioning by acetylcholinesterase antibodies. J Pharmacol Exp Ther 1996:277:817-22.

27 Brimijoin S, Hammond P, Khraibi AA, et al. Catecholamine release and excretion in rats with immunologically induced preganglionic sympathectomy. J Neurochem 1994;62:2195-204.

28 Cook JE, Kolka MA, Wenger CB. Chronic pyridostigmine bromide administration: side effects among soldiers working in a desert environment. Mil Med 1992;157:250-4.

29 Gemmill JD, Venables GS, Ewing DJ. Noradrenaline response to edrophonium in primary autonomic failure: distinction between central and peripheral damage. Lancet 1988;i:1018-21.

30 Stein RD, Backman SB, Collier B, et al. Bradycardia produced by pyridostigmine and physostigmine. Can J Anaesth 1997;44:1286-92.

31 Kolka MA, Stephenson LA. Human temperature regulation during exercise after oral pyridostigmine administration. Aviat Space Environ Med 1990;61:220-4

32 Nobrega AC, Carvalho AC, Santos KB, et al. Cholinergic stimulation with pyridostigmine blunts the cardiac responses to mental stress. Clin Auton Res 1999;9:11-16.

33 Wright RB. Myasthenia. In: Klawans HL, Goetz CG, Tanner CM, eds. Textbook of clinical neuropharmacology and therapeutics, 2nd ed. New York: Raven Press, 1992:505-16.

34 Finley JC, Pascuzzi RM. Rational therapy of myasthenia gravis. Semin Neurol 1990;10:70-82.

35 Camilleri M, Malagelada JR, Stanghellini V, et al. Gastrointestinal motility disturbances in patients with orthostatic hypotension. Gastroenterology 1985;88:1852-9.

36 Low PA, Bannister R. Multiple system atrophy and pure autonomic failure. In: Low PA, ed. Clinical autonomic disorders: evaluation and management, 2nd ed. Philadelphia: Lippincott-Raven, 1997:555-75.

37 Low PA, McLeod JG. Autonomic neuropathies. In: Low PA, ed. Clinical autonomic disorders: evaluation and management, 2 nd ed. Philadelphia: Lippincott-Raven, 1997:463-86.

38 Sadjadpour K. Pyridostigmine bromide and constipation in Parkinson's disease [letter]. JAMA 1983;249:1148. 